\title{
Erratum to: Differential regulation of protein phosphatase 1 (PP1) isoforms in human heart failure and atrial fibrillation
}

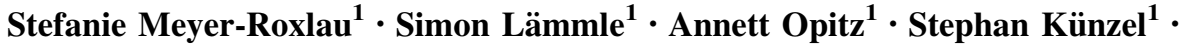 \\ Julius P. Joos ${ }^{1} \cdot$ Stefan Neef $^{2} \cdot$ Karolina Sekeres $^{1} \cdot$ Samuel Sossalla $^{2}$. \\ Friedrich Schöndube $^{3} \cdot$ Konstantin Alexiou $^{4} \cdot$ Lars S. Maier $^{2} \cdot$ Dobromir Dobrev $^{5}$. \\ Kaomei Guan ${ }^{1} \cdot$ Silvio Weber ${ }^{1} \cdot$ Ali El-Armouche ${ }^{1}$ (ID
}

Published online: 7 July 2017

(C) Springer-Verlag GmbH Germany 2017

\section{Erratum to: Basic Res Cardiol (2017) 112:43 \\ DOI 10.1007/s00395-017-0635-0}

Unfortunately, the author name Samuel Sossalla contained a typing error in the original publication. The original article has been corrected.

We apologize for any inconvenience caused.

The online version of the original article can be found under doi:10.1007/s00395-017-0635-0.

Silvio Weber

Silvio.Weber@tu-dresden.de

$\triangle$ Ali El-Armouche

Ali.El-Armouche@tu-dresden.de

1 Department of Pharmacology and Toxicology, Medical

Faculty, Technische Universität Dresden, Fetscherstraße 74,

Dresden 01307, Germany

2 Department of Internal Medicine II, University Hospital Regensburg, Regensburg, Germany

3 Department of Thoracic and Cardiovascular Surgery, University Medical Centre Goettingen, Göttingen, Germany

4 Department of Heart Surgery, Dresden University of Technology, Dresden, Germany

5 Institute of Pharmacology, West German Heart and Vascular Center, University Duisburg-Essen, Essen, Germany 